\title{
Hybrid TLBO and BFGS for structural health monitoring optimisation problems
}

\author{
Nantiwat Pholdee ${ }^{1, a}$ and Sujin Bureerat ${ }^{1, b^{*}}$ \\ ${ }^{1}$ Sustainable and Infrastructure Research and Development Center, Department of Mechanical \\ Engineering, Faculty of Engineering, Khon Kaen University, Thailand, 40002 \\ anantiwat@kku.ac.th, ${ }^{\mathrm{b}}$ sujbur@kku.ac.th
}

Keywords: Structural health monitoring, Meta-heuristics, Modal data, Damage detection

\begin{abstract}
This paper proposes hybrid teaching learning based optimisation (TLBO) and the Broyden-Fletcher-Goldfarb-Shanno algorithm (BFGS) for solving structural health monitoring optimisation problems. Two structural damage detection problems from two different truss structures are used to examine the search performance of the proposed algorithm while several well establish meta-heuristics (MHs) are used for benchmarking. The results indicated that the proposed algorithm is superior to the others. This study clarified that integrating BFGS into TLBO leads to increasing search performance of the new hybrid algorithm for solving structural health monitoring optimisation problems.
\end{abstract}

\section{Introduction}

In practical work in the structural engineering field, other than designing a structure to meet working function and optimisation, maintenance of the structure for safety under service loadings throughout its lifetime is also a major issue. In this regard, a structural inspection and/or damage detection technique to detect some undesirable occurrence such as defects in structural elements, cracks from fatigue, and wears is the most concerned. Although some damage detection techniques are recently available such as ultrasonic analysis and X-ray scan, the techniques are suitable for only small structures or elements while, for large structures, it is difficult to check thoroughly by these techniques. Therefore, an efficient structural damage detection technique which can identify the presence of structural damage, localising it, and assessing the severity [1] throughout a structure within one measurement is required.

The most popular damage detection technique relevant to the issue mentioned above is to use the changing of the structural model information such as natural frequencies and mode shapes. The main idea is to use the modal data of a normal structure as the baseline, if the modal data alert from normal values, the damage might occur. The used of modal data based damage detection can be from, using the modal data directly such as in references [2-5], applying soft computing such as fuzzy logic systems [6] and neural networks [7]. Recently, meta-heuristics (MHs) have been introduced to solve a structural damage detection inverse problem. The successful use of MHs for structural damage detection has been reported worldwide [8-12]. Nevertheless, in the field of meta-heuristics, there are no MHs which perform consistently over a wide range of problems, developing MH algorithms with fast convergence and high consistency is still challenging.

Therefore, this paper presents a hybridisation of teaching learning based optimisation (TLBO) and Broyden-Fletcher-Goldfarb-Shanno algorithm (BFGS) for structural damage detection. In this wor $\mathrm{k}$, two truss structures are used as numerical test problems to assess the performance of a number of MHs and the proposed algorithm for solving structural damage detection and localisation. The results obtained are compared and discussed.

\section{Formulation of Damage Detection based on Natural Frequency.}

In this study, damage detection of truss structure is investigated. Changing of structural natural 
frequencies is employed for damage localisation of the truss structure. The damaged location of structural can be found by updating the finite element model until the computed modal parameters and the measured modal parameters are equivalent. The finite element of undamped free vibration of the truss structure can be expressed as follow;

$$
[\mathbf{K}]\left\{\phi_{j}\right\}-\omega^{2}{ }_{j}[\mathbf{M}]\left\{\phi_{j}\right\}=0
$$

where, $[\mathbf{K}]$ and $[\mathbf{M}]$ are a structural stiffness matrix and structural mass matrix respectively while $\left\{\phi_{j}\right\}$ and $\omega_{j}$ are the $j^{\text {th }}$ mode shape and its coressposind natural frequency respectively. For non-damaged structure, $[\mathbf{K}]$ can be expressed as the summation or assembly of element stiffness matrices $\left[\mathbf{k}_{\mathrm{e}}\right]$,

$$
[\mathbf{K}]=\sum_{i=1}^{n_{e}}\left[\mathbf{k}_{e}\right]
$$

where, $i$ and $n_{\mathrm{e}}$ are the $i^{\text {th }}$ element and the total number of elements while [M] can be obtained in a similar fashion as with the stiffness matrix. For the damaged structure, the element stiffness matrix of the damaged structure can be written as percentage of damage in element structural, denoted by [ $\left.\mathbf{K}_{\mathrm{d}}\right]$, which can be calculated by

$$
\left[\mathbf{K}_{d}\right]=\sum_{i=1}^{n_{e}} \frac{100-p_{i}}{100}\left[\mathbf{k}_{e}\right]
$$

where, $p_{\mathrm{i}}$ is percentage of damage in the $i^{\text {th }}$ element.

To identify the damage of the structural elements, the optimisation problem is defined to minimise the root mean square error of computed natural frequencies and measured natural frequencies in the first $n_{\text {mode }}$ mode. The objective function is then defined as follows:

$$
\operatorname{Min}: f(\mathbf{x})=\sqrt{\frac{\sum_{j=1}^{n_{\text {made }}\left(\omega_{j, \text { damage }}-\omega_{j, \text { computed }}\right)^{2}}}{n_{\text {mode }}}}
$$

where, $\omega_{j \text {,damage }}$ and $\omega_{j, \text { computed }}$ are the structural natural frequency of mode $j$ obtained from a damaged structure and that from solving (1) -(3). The design variable (x) is the value of element damage percentages which can be express as $\mathbf{x}=\left\{p_{1}, \ldots, p_{\text {ne }}\right\}^{T}$. Note that the lowest five modes are used to compute the objecitve function in this study.

\section{Test Problems with Trusses}

To investigate the performance of the proposed algorithm, two damage detection problems derived from two truss structures are used in this study while several well established MHs are used to compare. For simplicity, damage of the structure will be simulated and the natural frequencies will be obtained from finite element analysis rather than performing real experimental modal analysis.

Nine-Bar Truss. Fig. 1a shown the schematic structure of the nine bar truss [8]. All bar element cross section areas of this structure are set to be $0.0025 \mathrm{~m}^{2}$. Material properties including young modulus and density are set to be $200 \mathrm{Gpa}$ and $7,850 \mathrm{~kg} / \mathrm{m} 3$, respectively. $50 \%$ damage at element number 2 is simulated as a damaged structure. The data of natural frequencies of the undamaged and damaged 9-bar structures are shown in Table 1.

SeventyTtwo-Bar Trus. Fig. 1b shown the schematic structure of the seventy-two bar truss [9]. For this structure, all bar element cross section areas of this structure are set to be $0.0025 \mathrm{~m}^{2}$ while 2270 $\mathrm{kg}$ non-structural mass is attached to the top four nodes. Material properties including young modulus and density are set to be $6.98 \times 10^{10}$ pa and $2,770 \mathrm{~kg} / \mathrm{m}^{3}$, respectively. $15 \%$ damage at element number 55 is simulated as damaged structure (15\% damage in element number 56,57 , or 58 will result in the same set of natural frequencies). The data of natural frequencies of the undamaged and damaged 72-bar truss structure are shown in Table 1. 
Table 1. Natural frequencies $(\mathrm{Hz})$ of damaged and undamaged of the structures.

\begin{tabular}{|c|c|c|c|c|}
\hline \multirow{2}{*}{ Modes } & \multicolumn{2}{|c|}{ 9 bar truss } & \multicolumn{2}{c|}{ 72 bar truss } \\
\cline { 2 - 5 } & Undamaged & 50\% damage at element number 2 & Undamaged & 15\% damage at element number 55 \\
\hline 1 & 38.3606 & 36.0103 & 6.0455 & 5.9553 \\
\hline 2 & 74.5226 & 66.3895 & 6.0455 & 6.0455 \\
\hline 3 & 117.8257 & 104.8556 & 10.4764 & 10.4764 \\
\hline 4 & 198.0133 & 194.2126 & 18.2297 & 18.1448 \\
\hline 5 & 260.1367 & 256.4372 & 25.4939 & 25.4903 \\
\hline
\end{tabular}

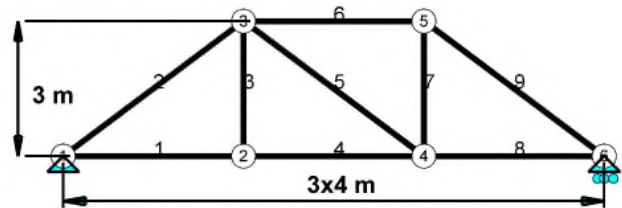

(a)

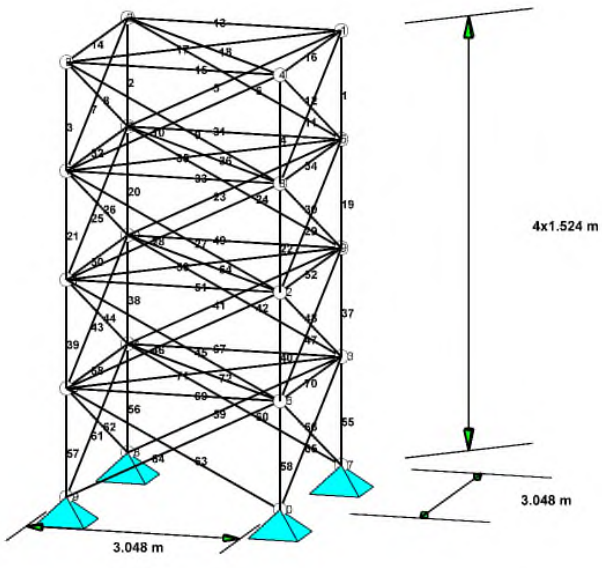

(b)

Fig. 1 a.) Nine bar truss b.) Seventy-two bar truss.

\section{Hybrid Teaching-Learning based Optimisation and Broyden-Fletcher-Goldfarb-Shanno Algorithm}

The teaching-learning based optimisation (TLBO) algorithm is an evolutionary algorithm or an optimiser without using function derivatives proposed by Rao et al. [13]. The search procedures starts with a randomly created initial population which is a group of design solutions. Learners are identical to design solutions whereas the best one is considered a teacher. The objective function is analogous to the knowledge which required to be improved towards the optimum solution. Having identified a teacher and other learners for the current iteration, the population will be updated by two stages including Teacher Phase and Learner Phase. In the Teacher Phase, an individual ( $\left.\mathbf{x}_{i}\right)$ will be updated based on the best individual ( $\left.\mathbf{x}_{\text {teacher }}\right)$ and the mean values of all population ( $\mathbf{x}_{\text {mean }}$ ) as follows:

$$
\mathbf{x}_{\text {new }, i}=\mathbf{x}_{\text {old }, i}+r\left\{\mathbf{x}_{\text {teacher }}-\left(T_{F} \cdot \mathbf{x}_{\text {mean }}\right)\right\}
$$

where, $T_{\mathrm{F}}$ is a teaching factor, which can be either 1 or 2 and $r \in[0,1]$ is a uniform random number.

For the "Learner Phase", the members in the current population will be modified by exchanging information between themselves. Two individuals $\mathbf{x}_{i}$ and $\mathbf{x}_{j}$ will be chosen at random, where $i \neq j$. The update of the solutions can then be calculated as:

$$
\mathbf{x}_{\mathrm{new}, i}= \begin{cases}\mathbf{x}_{\mathrm{old}, i}+r\left(\mathbf{x}_{i}-\mathbf{x}_{j}\right) & \text { if } f\left(\mathbf{x}_{i}\right)<f\left(\mathbf{x}_{j}\right) \\ \mathbf{x}_{\mathrm{old}, i}+r\left(\mathbf{x}_{j}-\mathbf{x}_{i}\right) & \text { if } f\left(\mathbf{x}_{j}\right)<f\left(\mathbf{x}_{i}\right)\end{cases}
$$

At both teacher and learner phases, the new solution $\left(\mathbf{x}_{\text {new }}\right)$ will replace its parent if it has better knowledge or produces better objective function value, otherwise, it will be rejected. The two phases are sequentially operated until the termination criterion is fulfilled. More detail of TLBO algorithm can be found in the reference [13].

For the hybrid of TLBO and BFGS (TLBO-BFGS), the general search procedures are similar to the original TLBO. However, after finishing the Teacher Phase, the binomial crossover technique is 
applied if a uniform random number generated from 0 to 1 (rand $\left.)_{1}\right)$ is lower than the crossover probability $\left(P_{\mathrm{c}}\right)$ as sohwn in the following equation:

$$
x_{\text {new }, j}\left\{\begin{array}{rc}
x_{\text {old }, j} & \text { if } \text { rand }_{2}<C R_{1} \quad j=1, \ldots, D \\
x_{\text {teacher }, j} & \text { if } C R_{1} \leq \text { rand }_{2}<C R_{2} \quad j=1, \ldots, D
\end{array}\right.
$$

where, $\operatorname{rand}_{2}$ is a uniform random number generated from 0 to 1 . The $C R_{1}$ and $C R_{2}$ are the predefined crossover rates while $D$ is the number of design variables, respectively. Then, the Learner Phase is activated. BFGS is used to seach local optimum for a number of function evaluations $\left(n_{\mathrm{BFGS}}\right)$ after finishing the Learner Phare, if a random number generated from 0 to 1 ( rand $_{3}$ ) is lower than a predefined probbability $\left(P_{\mathrm{BFGS}}\right)$. The computation step of the (TLBO-BFGS) is shown in Algorithm 1. Note that, the BFGS used in this study is a quasi-newton based BFGS which MATLAB toolbox function named fminunc is used. The gradient is computed by means of the finite different method.

Algorithm 1. TLBO-BFGS.

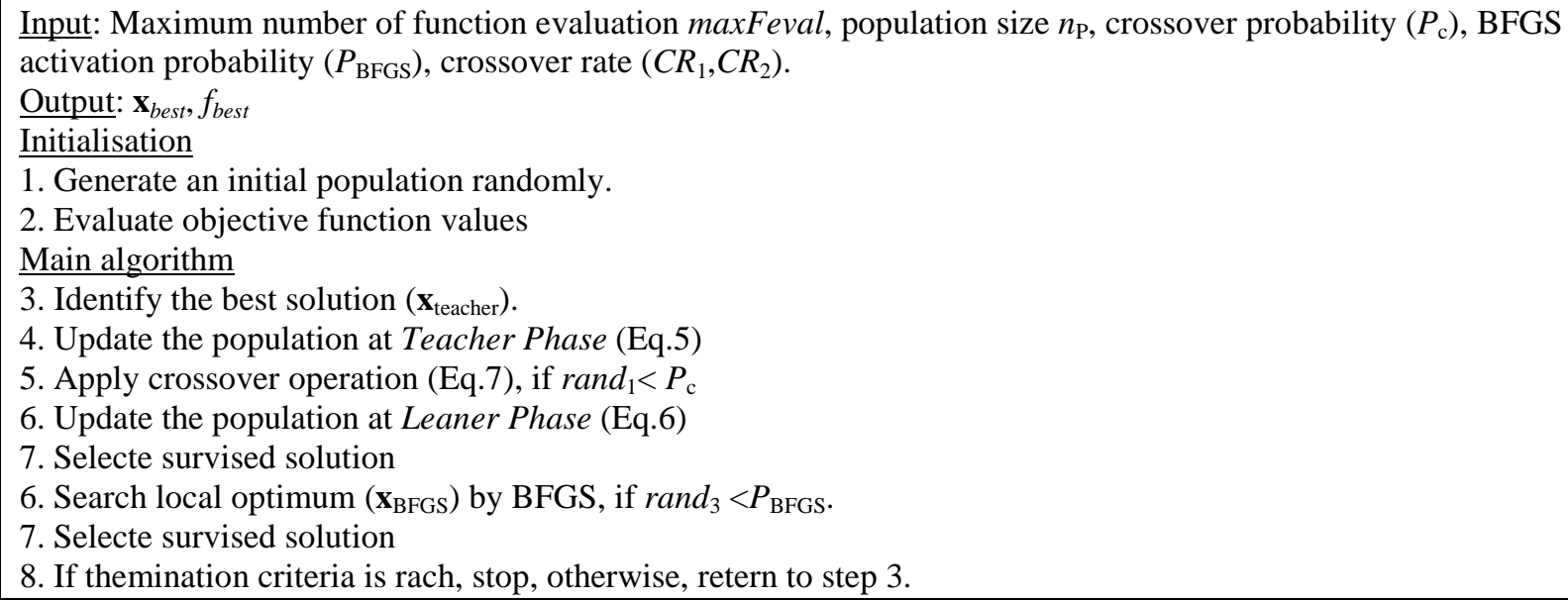

\section{Numerical Experiment}

To verified the search performance of the proposed TLBO-BFGS, several MHs are compared based on solving the truss damage detection problems. The MHs and their optimisation parameter settings used in this study (details of notations can be found in the corresponding references of each method) are detailed as [14]:

1. Differential evolution (DE) [15]: a DE/best/2/bin strategy was used. A scaling factor, crossover rate and probability of choosing elements of mutant vectors are $0.5,0.7$, and 0.8 respectively.

2. Real-code ant colony optimisation (ACOR) [8]: The parameter settings are $\mathrm{q}=0.2$, and $\xi=1$.

3. Particle swarm optimisation (PSO) [16]: The starting inertia weight, ending inertia weight, cognitive learning factor, and social learning factor are assigned as $0.5,0.01,0.5$ and 0.5 respectively.

4. Evolution strategies (ES) [17]: The algorithm uses a binary tournament selection operator and a simple mutation without the effect of rotation angles.

5. Teaching-learning-based optimisation (TLBO) [13]: Parameter settings are not required.

6. TLBO-BFGS (Algorithm 1): The $P_{\mathrm{c}}, P_{\mathrm{BFGS}}, C R_{1}$, and $C R_{2}$ are set to be $0.3,0.15,0.25$, and 0.5 , respectively, while the $n_{\mathrm{BGGS}}$ is set to be $10 n_{\mathrm{p}}$.

Each optimiser is used to solve each problem for 30 optimisation runs. The population size is set to be 15 and 35 for the 9-bar and 72-bar trusses respectively whereas the number of iterations is 200 for all case studies. For the optimisers using different population sizes, their search processes are terminated with the total number of functions evaluations (FEs) equal to $15 \times 200$, and $35 \times 200$ for the 9-bar and 72-bar trusses respectively. Another termination criterion is when one of the members in the current population has an objective function value as $1 \times 10^{-3}$. 


\section{Results and Discussion}

After solving the truss damage detection problems for 30 optimisation runs, the results are illustrated in Table 2-3. The mean and standard deviation (STD) values of the objective function are used to measure the search convergence and consistency of the algorithms.

For the results of the 9-bar truss with 5\% damage at element 2 in Table 2, the best performer based on the objective mean values is TLBO-BFGS while the second best and the third best are ACOR and DE, respectively. Based on STD, the best performer is ACOR while the second best is TLBO-BFGS. The minimum objective function value is obtained from using TLBO-BFGS. For this case, the optimisers which can detect the damage of structure for 30 times from 30 runs are ACOR and TLBO-BFGS The most efficient algorithm is TLBO-BFGS which obtained the results with the average of 581 function evaluations.

For the 72-bar truss with $15 \%$ damage at element number 55, the results are given in Table 3 . The best performer based on mean of objective function values is TLBO-BFGS while the second best and the third best are TLBO and ACOR respectively. Based on the STD values, the best performer is TLBO-BFGS while the second and third best are ACOR and TLBO respectively. The minimum objective function value is obtained by the TLBO-BFGS. For this case, only TLBO-BFGS can detect the damage of structure for 30 times from 30 runs which using the lowest average number of function evaluation of 3,085 to obtained the results.

Overall, it was found that integrating crossover operation and BFGS into the TLBO can improve its search convergence and search consistency. The hybrid version is found to be the best performer for all cases.

Table 2. Results for 9 bar truss with 50\% damage at element 2.

\begin{tabular}{|c|c|c|c|c|c|c|}
\hline \multirow{2}{*}{ Optimisers } & \multicolumn{4}{|c|}{ Objective function Values } & \multirow{2}{*}{$\begin{array}{c}\text { O. of successful runs } \\
\text { from 30 runs }\end{array}$} & \multirow{2}{*}{ Mean of FEs } \\
\cline { 2 - 5 } & Mean & STD & Min & Max & 29 & 859 \\
\hline DE & 0.0035 & 0.0146 & 0.0005 & 0.0807 & $\mathbf{3 0}$ & $\mathbf{1 0 7 8}$ \\
\hline ACOR & 0.0008 & $\mathbf{0 . 0 0 0 1}$ & 0.0005 & 0.0010 & 4 & 2670 \\
\hline TLBO & 1.2033 & 1.4272 & 0.0003 & 4.1611 & 0 & 3000 \\
\hline ES & 1.2596 & 1.1083 & 0.0097 & 4.1785 & 0 & 3000 \\
\hline PSO & 9.1627 & 2.7134 & 3.0601 & 13.6549 & $\mathbf{3 0}$ & $\mathbf{5 8 1}$ \\
\hline TLBO-BFGS & $\mathbf{0 . 0 0 0 3}$ & 0.0003 & $\mathbf{0 . 0 0 0 0}$ & 0.0010 & & \\
\hline
\end{tabular}

Table 3. Results for 72 bar truss with $15 \%$ damage at element number 55.

\begin{tabular}{|c|c|c|c|c|c|c|}
\hline \multirow{2}{*}{ Optimisers } & \multicolumn{4}{|c|}{ Objective function Values } & \multirow{2}{*}{$\begin{array}{c}\text { No. of successful runs } \\
\text { from 30 runs }\end{array}$} & \multirow{2}{*}{ Mean of FEs } \\
\cline { 2 - 5 } & Mean & STD & Min & Max & 2 & 6990 \\
\hline DE & 0.0370 & 0.0663 & 0.0008 & 0.2940 & 2 & 7000 \\
\hline ACOR & 0.0059 & 0.0011 & 0.0040 & 0.0089 & 0 & 4218 \\
\hline TLBO & 0.0014 & 0.0012 & $\mathbf{0 . 0 0 0 5}$ & 0.0058 & 23 & 6869 \\
\hline ES & 0.0075 & 0.0122 & 0.0009 & 0.0667 & 9 & 7000 \\
\hline PSO & 1.8459 & 0.1700 & 1.4582 & 2.1727 & 0 & $\mathbf{3 0 8 5}$ \\
\hline TLBO-BFGS & $\mathbf{0 . 0 0 0 9}$ & $\mathbf{0 . 0 0 0 1}$ & $\mathbf{0 . 0 0 0 6}$ & 0.0010 & $\mathbf{3 0}$ & \\
\hline
\end{tabular}

\section{Conclusions}

Hybridising of BFGS and TLBO is presented for truss structural damage detection problems in this work. Two structural damage detection test problems are used to examine the search performance of several well established MHs and the proposed algorithm. It is found that the proposed hybrid TLBO-BFGS is superior to the rest for this task. Integrating the binomial crossover operation and BFGS into the TLBO leads to the more powerful $\mathrm{MH}$ for both convergence rate and search consistency. 


\section{Acknowledgement}

The authors are grateful for the support from the Thailand Research Fund (TRF).

\section{References}

[1] J. J. Sinou, A review of damage detection and health monitoring of mechanical systems from changes in the measurement of linear and non-linear vibrations, In: Sapri. RC, editor. Mechanical Vibrations: Measurement, Effects and Control: Nova Science Publishers, Inc., 2009. pp. 643-702.

[2] J. M. Lifshitz, A. Rotem, Determination of Reinforcement Unbonding of Composites by a Vibration Technique, J. Compos. Mater. 3 (1969) 412-423.

[3] G. Hearn, R. B. Testa, Modal Analysis for Damage Detection in Structures, J. Struc. Eng. 117 (1991) 3042-3063.

[4] A. Messina, E. J. Williams, T. Contursi, Structural Damage Detection By A Sensitivity And Statistical-Based Method, J. Sound Vib. 216 (1998) 791-808.

[5] B. H. Koh, S. J. Dyke, Structural health monitoring for flexible bridge structures using correlation and sensitivity of modal data, Comput. Struct. 85 (2007) 117-130.

[6] M. Wang, Y. T. Feng, C. Y. Wang, Numerical investigation of initiation and propagation of hydraulic fracture using the coupled Bonded Particle-Lattice Boltzmann Method, Comput. Struct. (2016), In press.

[7] O. Abdeljaber, O. Avci, Nonparametric Structural Damage Detection Algorithm for Ambient Vibration Response: Utilizing Artificial Neural Networks and Self-Organizing Maps, J. Arch. Eng. 22 (2016) 04016004.

[8] A. Majumdar, D. K. Maiti, D. Maity, Damage assessment of truss structures from changes in natural frequencies using ant colony optimization, Appl. Math. Comput. 218 (2012) 9759-9772.

[9] A. Kaveh, A. Zolghadr, An improved CSS for damage detection of truss structures using changes in natural frequencies and mode shapes, Adv. Eng. Softw. 80 (2015) 93-100.

[10] J. Pal, S. Banerjee, A combined modal strain energy and particle swarm optimization for health monitoring of structures, J. Civil Struct. Health. Monit. 5 (2015) 353-363.

[11]Z. H. Ding, M. Huang, Z. R. Lu, Structural damage detection using artificial bee colony algorithm with hybrid search strategy, Swarm and Evolutionary Computation. 28 (2016) 1-13.

[12]H. Xu, Z. Ding, Z. Lu, J. Liu, Structural damage detection based on Chaotic Artificial Bee Colony algorithm. Struct, Eng. Mech. 55 (2015) 1223-1239.

[13]R. V. Rao, V. J. Savsani, D. P. Vakharia, Teaching-learning-based optimization: A novel method for constrained mechanical design optimization problems, Comput. Aided Design. 43 (2011) 303-315.

[14]N. Pholdee, S. Bureerat, Structural health monitoring through meta-heuristics - comparative performance study, Advances in Computational Design, An International Journal. (2016) accepted.

[15]R. Storn, K. Price, Differential Evolution - A Simple and Efficient Heuristic for global Optimization over Continuous Spaces, J. Global Optim. 11 (1997) 341-359.

[16]G. Venter, J. Sobieszczanski-Sobieski, Particle swarm optimization. AIAA Journal. 41 (2003) 1583-1589.

[17]T. Back, Evolutionary Algorithms in Theory and Practice. Oxford: Oxford University Press; 1996. 